LA WAENCE LIWEAMCAE WATIONAL LABOAAT OAY

\title{
Coherent communications, Imaging and Targeting
}

Eddy A. Stappaerts, Kevin L. Baker, Donald T. Gavel, Scott C. Wilks, Scot S. Olivier and James M. Brase

\section{October 10, 2003}

\author{
2004 IEEE Aerospace Conference \\ Big Sky, Montana \\ March 6-13, 2004
}

This document was prepared as an account of work sponsored by an agency of the United States Government. Neither the United States Government nor the University of California nor any of their employees, makes any warranty, express or implied, or assumes any legal liability or responsibility for the accuracy, completeness, or usefulness of any information, apparatus, product, or process disclosed, or represents that its use would not infringe privately owned rights. Reference herein to any specific commercial product, process, or service by trade name, trademark, manufacturer, or otherwise, does not necessarily constitute or imply its endorsement, recommendation, or favoring by the United States Government or the University of California. The views and opinions of authors expressed herein do not necessarily state or reflect those of the United States Government or the University of California, and shall not be used for advertising or product endorsement purposes.

This work was performed under the auspices of the U.S. Department of Energy by University of California, Lawrence Livermore National Laboratory under Contract W-7405-Eng-48. 


\title{
Coherent Communications, Imaging and Targeting ${ }^{1,2}$
}

\author{
Eddy Stappaerts, Kevin Baker, Don Gavel, S. Wilks, S. Olivier and J. Brase \\ Lawrence Livermore National Laboratory \\ 7000 East Ave \\ Livermore, CA \\ (925) 422-7307 \\ Stappaerts1@1lnl.gov
}

\begin{abstract}
Laboratory and field demonstration results obtained as part of the DARPA-sponsored Coherent Communications, Imaging and Targeting (CCIT) program are reviewed. The CCIT concept uses a Phase Conjugation Engine based on a quadrature receiver array, a hologram processor and a spatial light modulator (SLM) for highspeed, digital beam control. Progress on the enabling MEMS SLM, being developed by a consortium consisting of LLNL, academic institutions and small businesses, is presented.
\end{abstract}

\section{TABLE OF CONTENTS}

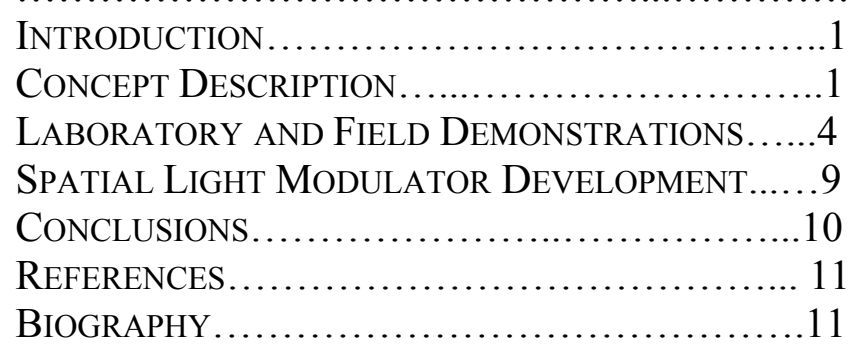

\section{INTRODUCTION}

The CCIT program is developing new capabilities in secure, very high data rate free-space communications, aberration-compensated imaging and sensing, and targeting at very long ranges. It combines innovative concepts and integration of MEMS SLMs, which provide digital wavefront control, with photonics and high-speed electronics. A team consisting of the Lawrence Livermore National Laboratory, academic institutions, small businesses, and aerospace companies has carried out Phase-I of the program, which was completed in July 2003 (SLM development continues). The SLMs being developed have numerous applications in addition to their use in the holographic concept discussed in this paper. The CCIT concept incorporates elements of the Active Tracking System developed by Northrop Grumman under DARPA sponsorship [1].

\section{CONCEPT DESCRIPTION}

The holographic concept is based on a Phase Conjugation Engine (PCE) that is common to all applications. As shown in Fig.1, it consists of a coherent receiver (focal plane array, charge-coupled device), a hologram processor, and an SLM. The PCE interfaces with other system modules according to the generic architecture of Fig. 2. Part of the input field is split off and directed to a high-speed detector to provide a trigger signal for a local oscillator (LO). The remainder is interfered with a pair of LO beams that are 90 degrees out of phase with one another to form quadrature holograms. The holograms are read-out and processed, and the conjugate of the extracted phase is applied to the SLM. The calculation of phase at any pixel is independent of other pixels, allowing very high frame rates by parallel, pipelined processing. The latency time is the sum of the hologram $\mathrm{read} / \mathrm{processing} /$ write time and the SLM response time. For a $128 \times 128$ FPA/SLM and a $25 \mathrm{MHz}$ clock, the $\mathrm{read} /$ processing/write time is about $5 \mu \mathrm{s}$. For a $10 \mu \mathrm{s}$ SLM response time, the program specification, the minimum latency time is therefore $\sim 15 \mu \mathrm{s}$. The holograms are also read into computer memory for application-specific processing, including system control and application algorithms.

\footnotetext{
0-7803-8155-6/04/\$17.00@2004 IEEE

${ }^{2}$ IEEEAC paper \#
} 


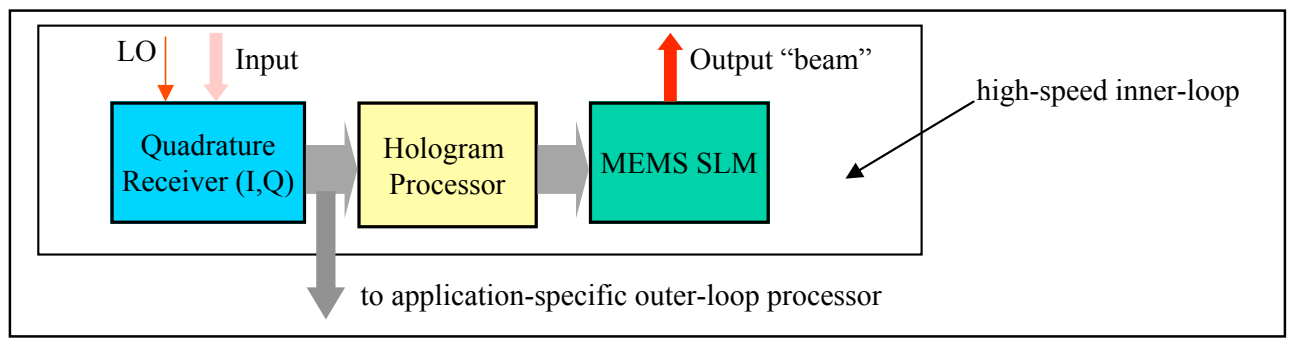

Figure 1. Phase Conjugation Engine schematic

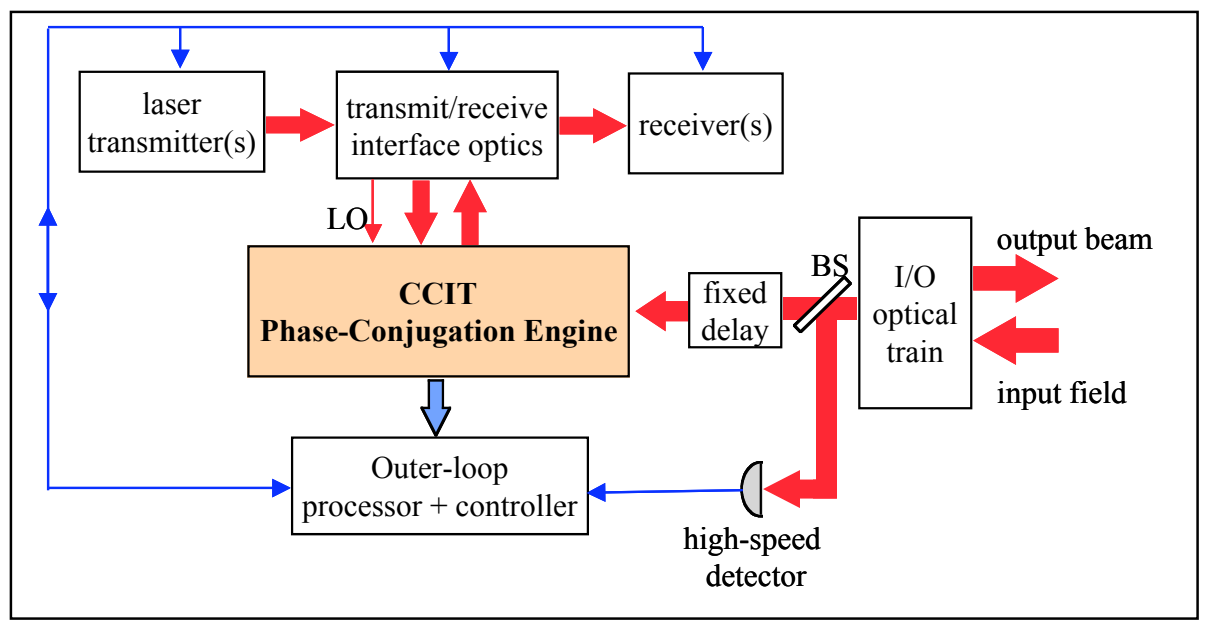

Figure 2. Generic system diagram

Operation will be described for communications from platform-A to platform-B (called "A" and "B" below). Both compensated receiver and conjugated transmitter configurations [2] will be described. Compensated receivers correct wavefronts similar to conventional adaptive optics, while conjugated transmitters apply a pre-correction to the transmitted beam. In some cases, the two configurations can be combined for optimum performance.

The concept uses separate lasers for acquisition/pointingand-tracking and for communications. The first function uses a short-pulse, on the order of nanoseconds, low-energy laser to provide stable holograms. Its repetition-rate, for atmospheric propagation, is typically $1-10 \mathrm{kHz}$.

Communications uses conventional telecom hardware.

The PCE has a field-of-view FOV $\sim(\mathrm{N} \lambda / \mathrm{D}) / 2^{\mathrm{b}-1}$, where $\lambda$ is the wavelength, $D$ the aperture size, $N$ the number of pixels per row, and $b$ the number of phase bits. Thus, for 1-bit operation, the FOV is about $\mathrm{N}$ times the diffraction-limit. The SLM is used as a programmable diffractive optic with theoretical diffraction efficiency of:

$$
\eta_{d}=\left[\frac{\sin (\pi / M)}{\pi / M}\right]^{2}
$$

where $\mathrm{M}=2^{\mathrm{b}}$. The efficiency is $40 \%, 81 \%, 95 \%$ and $99 \%$ for 1, 2, 3 and 4-bit operation, respectively.

\section{Compensated receiver}

Acquisition/link-closure starts by "A" transmitting a beacon beam towards " $\mathrm{B}$ ". The beam divergence is selected based on:

- Uncertainty about platform "B" direction,

- Uncertainty about platform "A" attitude angles,

- Trade-off with complexity of "A" coarse pointing-and-tracking (P\&T) system,

- Coherent-receiver signal-to-noise ratio at "B".

The conjugate wavefront of the beam received at " $\mathrm{B}$ " is applied to SLM-B. A communications beam is then transmitted from "A" to "B". The beam received at "B", which must be within the FOV of PCE-B, is reflected off of SLM-B, correcting the distortions on the received wavefront. The corrected beam is then directed to a telecom receiver.

Figure 3 shows a compensated receiver schematic. A polarizing beamsplitter, PBS1, and a beamsplitter, BS1, direct the received beacon beam towards its coherent 
receiver. A quarter-wave-plate in the LO path creates a circularly polarized beam containing two orthogonally polarized components 90 ninety degrees out of phase with one another. The part of the input beam reflected off of BS1, and the LO beam transmitted through BS1, both pass through a half-wave-plate that rotates polarization over 45 degrees, resulting in both beams having $\mathrm{p}$ and $\mathrm{s}$ components. A Wollaston prism separates the polarization components, resulting in quadrature holograms placed next to each other on the FPA.

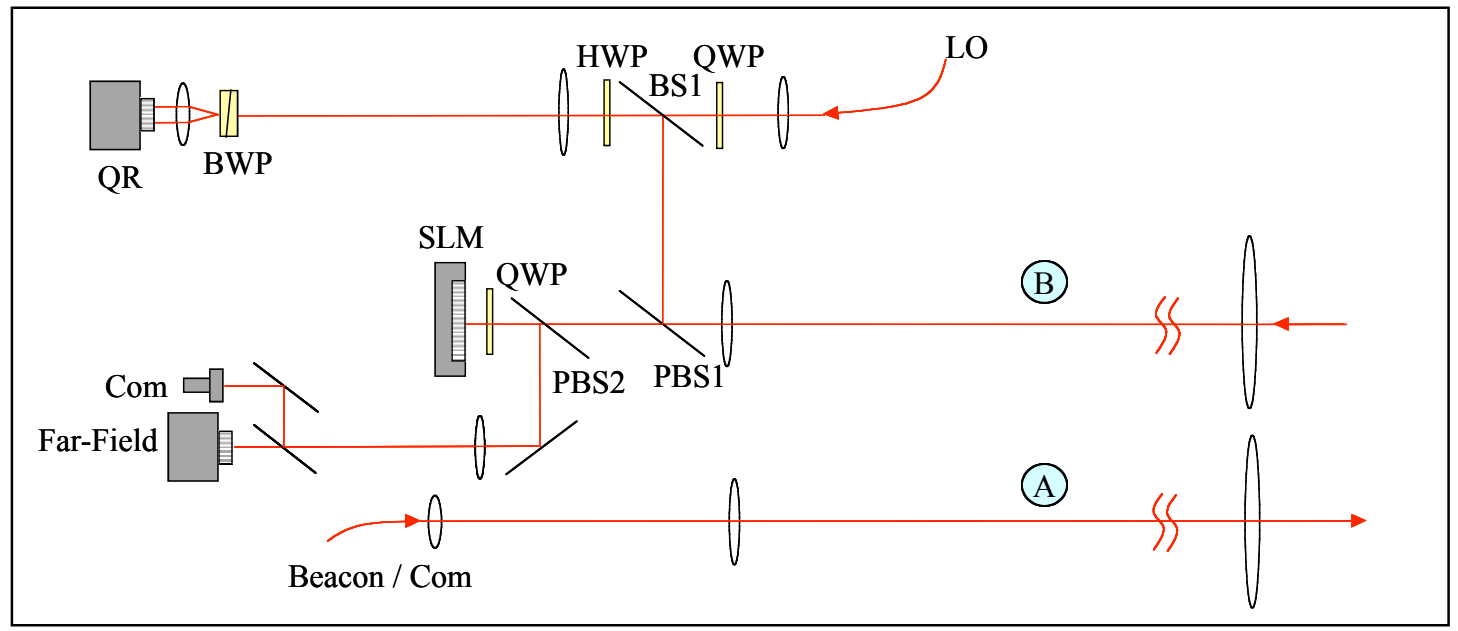

Figure 3. Compensated-receiver configuration

\section{Conjugated transmitter}

Acquisition/link-closure starts by " $\mathrm{B}$ " transmitting a beacon beam towards " $\mathrm{A}$ ". The beam is received and the conjugate wavefront applied to SLM-A. The communications beam is then reflected off of SLM-A, resulting in a beam, precorrected for distortions along the " $A$ " to " $B$ " path, transmitted towards " $\mathrm{B}$ ". The beam received at " $\mathrm{B}$ " is directed to a telecom receiver.

Figure 4 shows a conjugated transmitter schematic. Hologram write/read is similar to the compensated receiver. The communications beam is coupled into the optical train at PBS-2, reflects off of the SLM, and is transmitted.

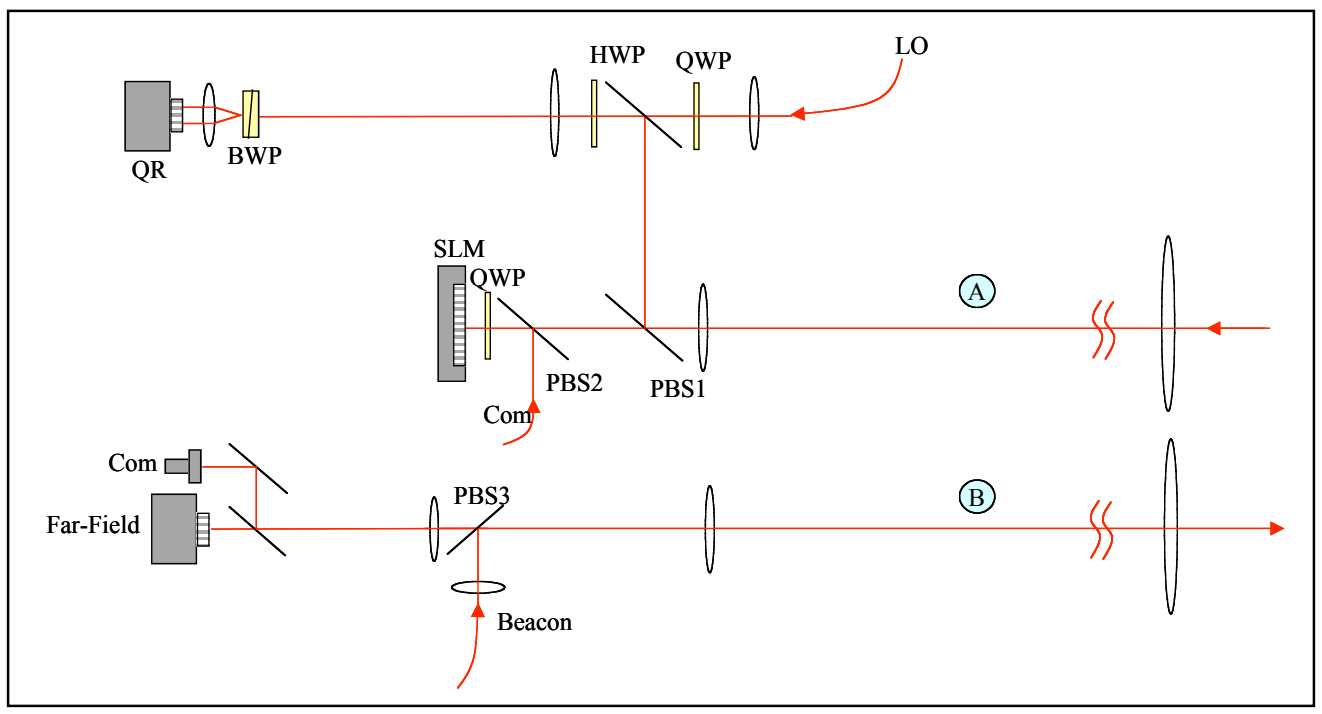

Figure 4. Conjugated transmitter configuration 


\section{LABORATORY AND FIELD DEMONSTRATIONS}

Demonstrations were performed at a wavelength of $0.53 \mu \mathrm{m}$ in the laboratory and at $1.53 \mu \mathrm{m}$ in the laboratory and the field. The visible-wavelength experiments investigated both compensated receiver and conjugated transmitter configurations. The results, in close agreement with theory, are being reported in a separate paper (Optics Letters). The present paper discusses the near-IR experiments. Linkclosure and atmospheric correction were characterized in detail and compared with theoretical projections.

Communications tests along the link have not yet been carried out with the near-IR system.
Figure 5 shows a schematic of the experimental setup. The coherent receiver used (part of) a commercial Indigo

Phoenix camera with $320 \times 256$ pixels. The SLM was a $32 \times$ 32 Si MEMS device developed by Boston MicroMachines Corporation and described below. The beacon and LO laser beams were produced by a fiber laser master-

oscillator/power-amplifier (MOPA) developed by HRL

Laboratories. A schematic and photograph are shown in Fig. 6 and 7, respectively. The device has two arms that provide the LO and beacon beam, respectively. The beacon and LO energies are about $7 \mu \mathrm{J}$ and $0.1 \mu \mathrm{J}$, respectively, within a pulse length of $1 \mathrm{~ns}$, at a repetition-rate of $1 \mathrm{kHz}$. The beam quality is near-diffraction-limited, $\mathrm{M}^{2} \sim 1.1$, and the pulse bandwidth is near-transform-limited.

\section{Laboratory demonstrations}

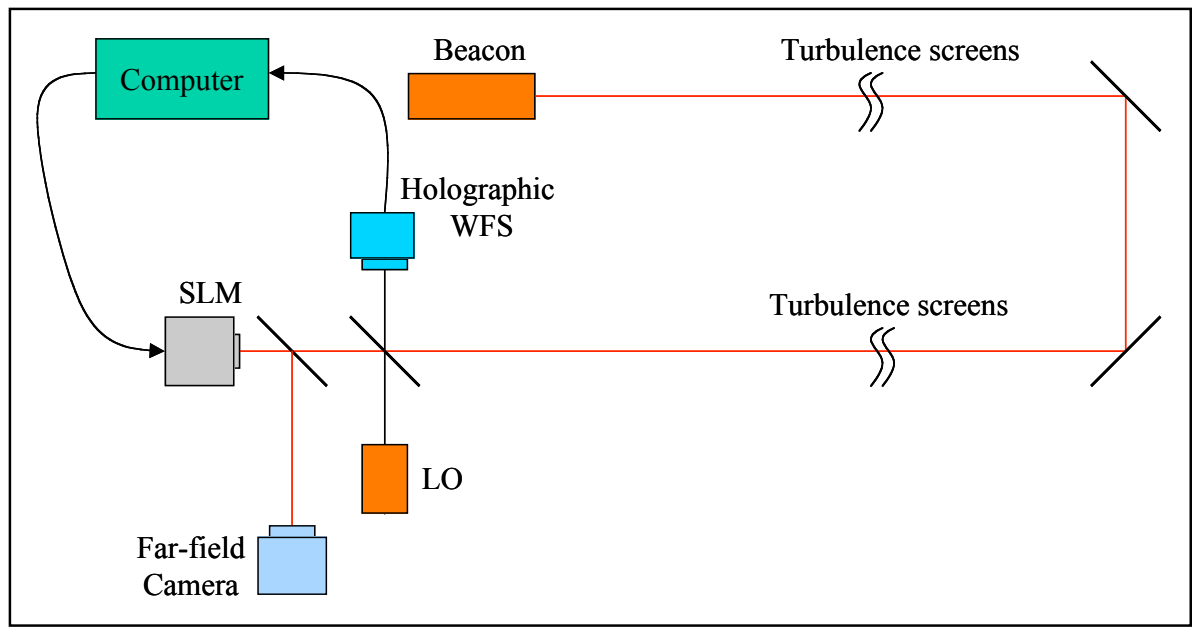

Figure 5. Schematic of the laboratory (and field test) breadboard

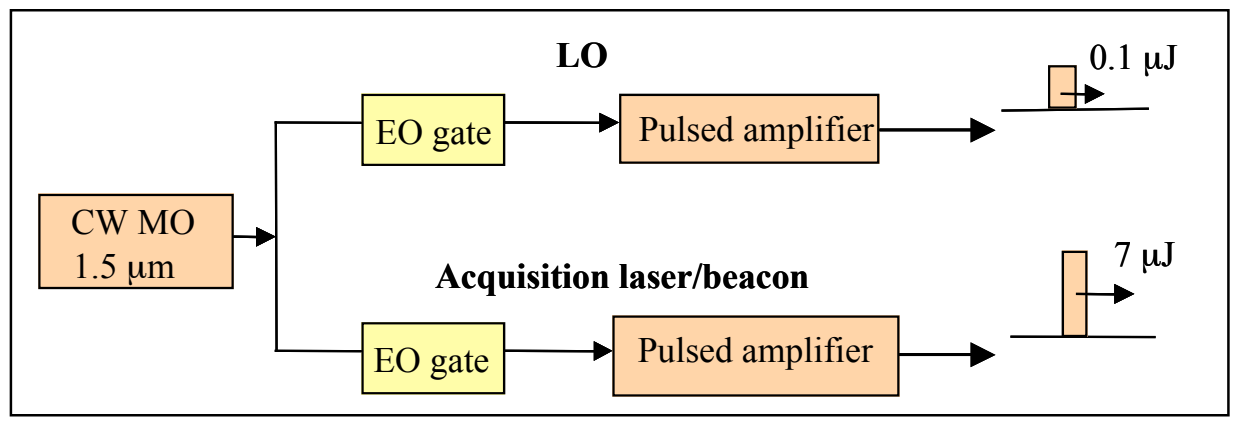

Figure 6. Block diagram of fiber laser MOPA developed by HRL Laboratories 


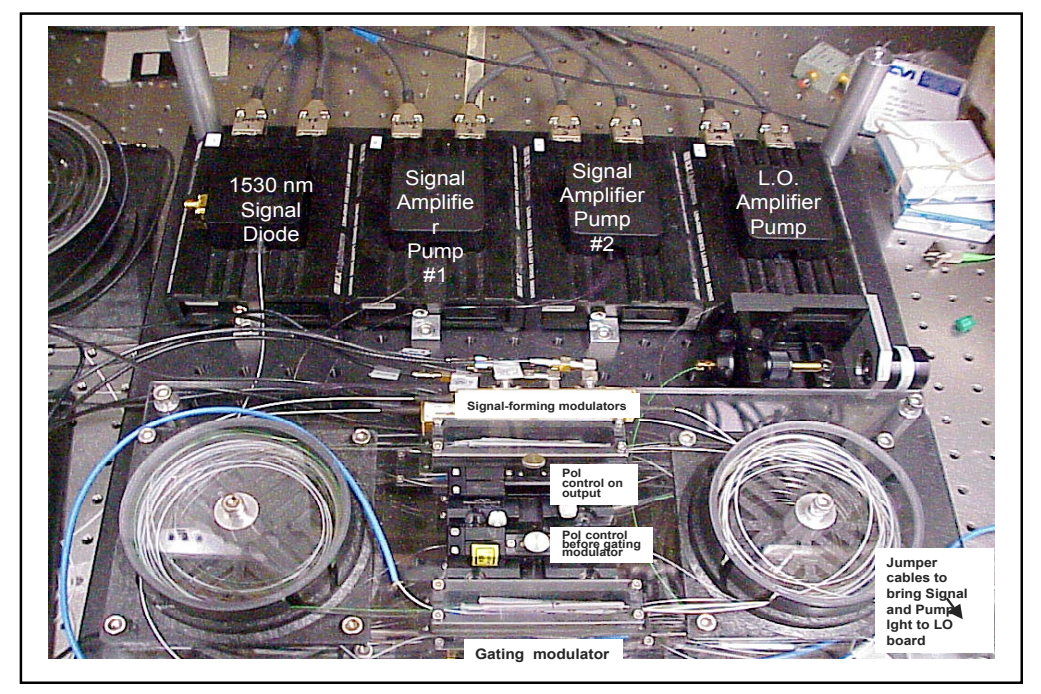

Figure 7. Photograph of fiber-laser MOPA developed by HRL Laboratories

Turbulence screens - Kolmogorov phase screens representative of the field test propagation path were designed and fabricated at LLNL. The plates were characterized both by measuring the phase structure function and by measuring the far-field pattern. For the phase structure measurements, $\sim 3000$ holograms of the beam transmitted through the phase plates were recorded.
Unwrapped phase profiles were extracted and from those the Fried parameter, $\mathrm{r}_{0}$, was obtained. Figure 8 shows the theoretical phase structure function, $\mathrm{D} \phi(\mathrm{r})=6.88\left(\mathrm{r} / \mathrm{r}_{0}\right)^{5 / 3}[3]$ and the experimental data. An analytic fit to the data yielded $\mathrm{r}_{0} \sim 1.3 \mathrm{~mm}$.

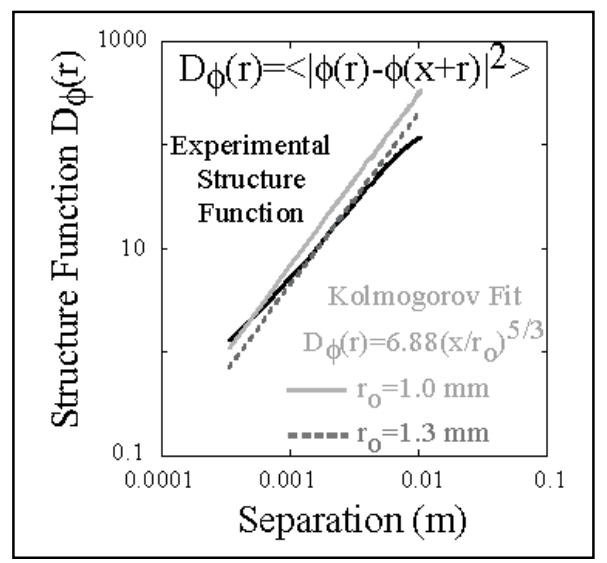

Figure 8. Theoretical and experimental phase-structure functions

The Fried parameter was also determined from far-field patterns. The resulting value was $\mathrm{r}_{0}=1.1 \mathrm{~mm}$, in good agreement with the $1.3 \mathrm{~mm}$ value obtained using the structure function. The results, in good agreement with theory, will be presented in a separate paper.

Phase power spectrum - Temporal waveforms of the phase fluctuations were obtained from the holograms by recording phase versus time for each of the pixels, at a $580 \mathrm{~Hz}$ acquisition frequency. Power spectra were obtained by Fourier- transforming and averaging the square of the transform modulus. Data were obtained at several screen rotation rates and fitted to $W_{\phi}(f)=\pi^{-1} F_{g}^{5 / 3} \mathrm{f}^{-8 / 3}$, where $F_{g}=$ $0.43 \mathrm{~V}_{\mathrm{t}} / \mathrm{r}_{0}$ is the Greenwood frequency [4]. Figure 9 shows measured and theoretical power spectra. Good agreement was obtained over several orders of magnitude. 


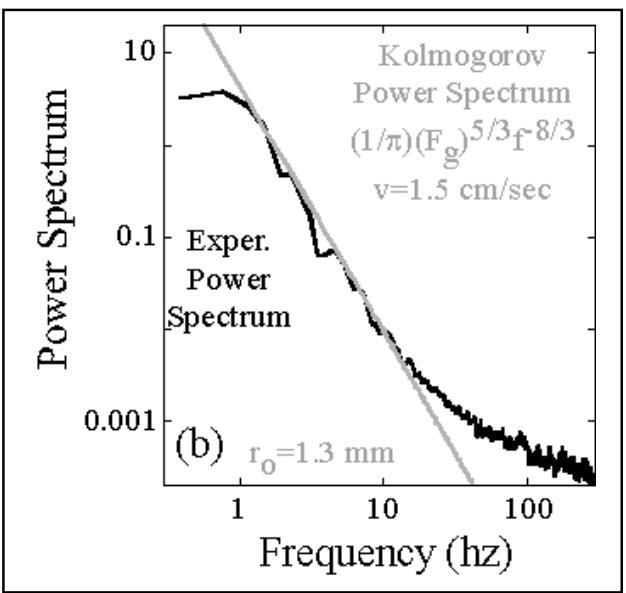

Figure 9. Phase power spectra for screen velocity of $1.5 \mathrm{~cm} / \mathrm{s}$

Measurements were also performed on Strehl ratio versus delay between hologram-write and SLM readout. The results, in good agreement with theory, will be presented in a separate paper.
Electronics and software - Figure 10 shows a diagram of the electronics/processing architecture. The system latency time was $\sim 1 \mathrm{~ms}$, and the frame rate $\sim 600 \mathrm{~Hz}$. Commercial electronics was used. Custom software was developed by LLNL. Details will be provided in a separate paper.

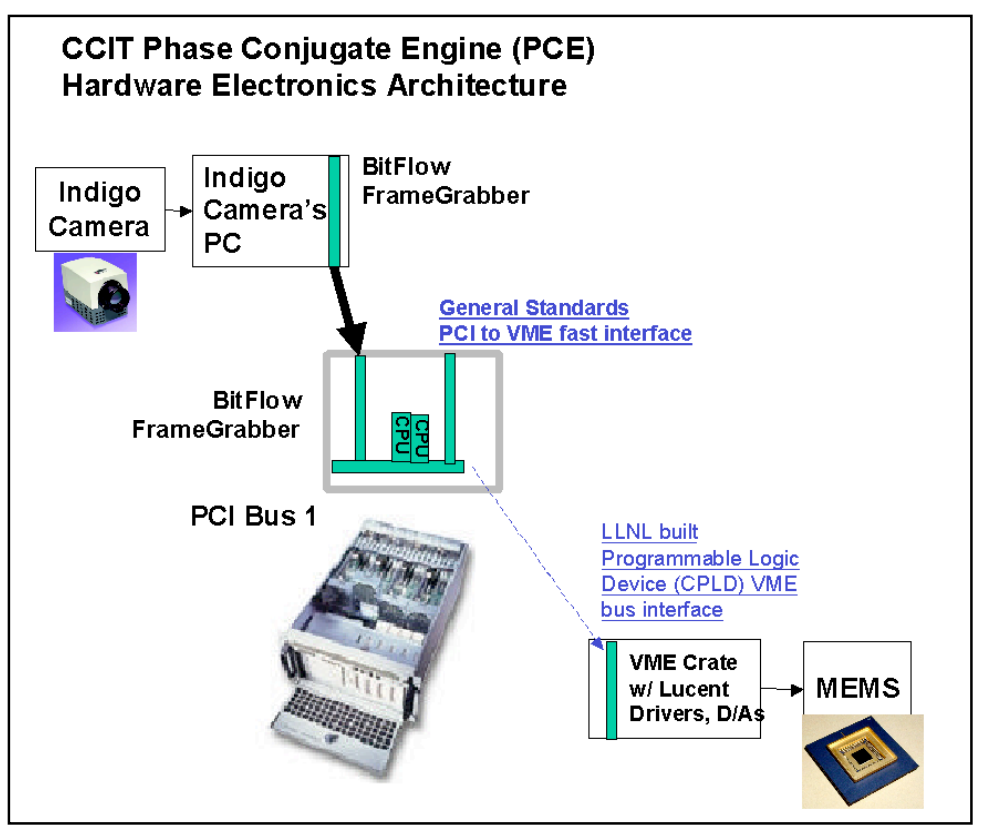

Figure10. Electronics/processing architecture

Experimental results - Aberration correction was quantified by measuring the Strehl ratio. The ratio was derived from measured and computed far-field patterns. The computed pattern used the scintillated near-field amplitude, as measured with the coherent receiver, and a flat phase-front. Strehl was defined as the ratio of the peak intensities. Its value was also compared with values projected for a Kolmogorov spectrum. Figure 11 shows uncorrected and corrected far-field patterns, while Fig. 12 shows far-field patterns with corresponding horizontal and vertical cross sections. Figure 13 shows absolute Strehl versus time. The scintillation index, $\left\{\sigma_{\mathrm{I}} / \mathrm{m}_{\mathrm{I}}\right)^{2}$, where $\sigma_{\mathrm{I}}$ and $\mathrm{m}_{\mathrm{I}}$ are the intensity standard deviation and mean, respectively, was 0.47 . 


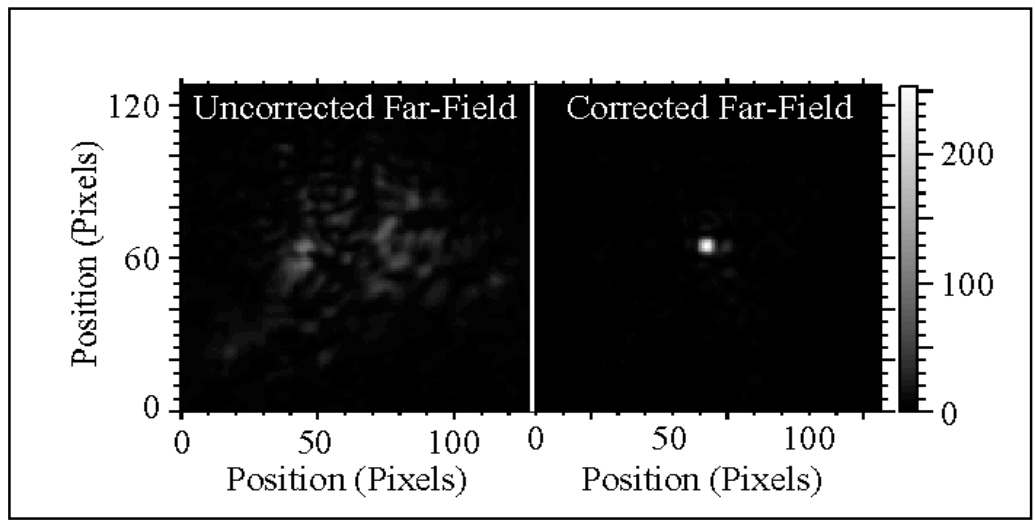

Figure 11. Uncorrected and corrected far-field patterns

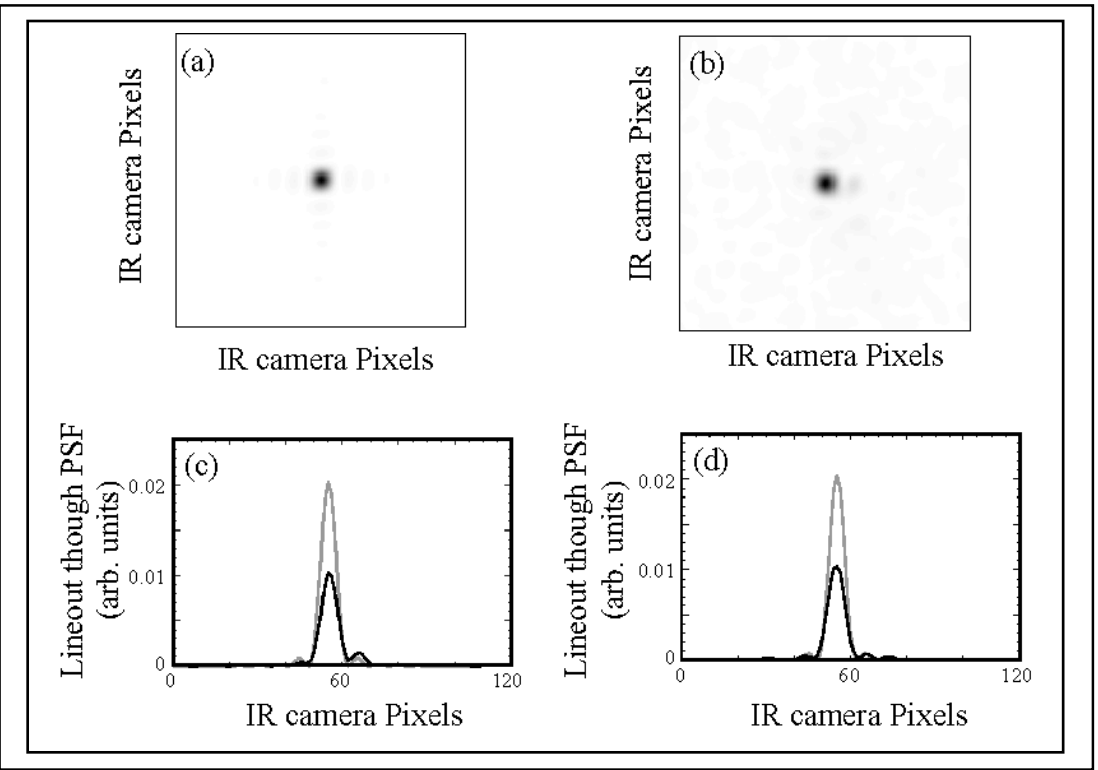

Figure 12. Measured and calculated far-field patterns

Top-left: ideal, top-right: measured. Bottom: lineout along horizontal and vertical directions

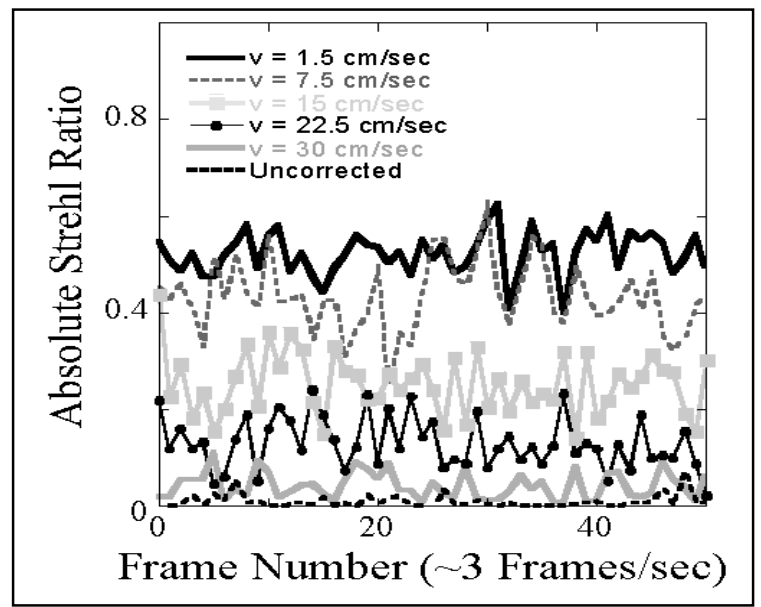

Figure 13. Uncorrected/corrected beam Strehl ratios for several phase screens velocities 
The achievable Strehl ratio was limited by a fitting error due to the finite number of SLM pixels, and by a less-than-unity area fill-factor. For a Kolmogorov spectrum, the fitting-error Strehl is given by $\exp \left[-1.3\left(\mathrm{~d} / \mathrm{r}_{0}\right)^{5 / 3}\right]=0.84$, where $\mathrm{d}$ is the pixel size [5]. The area fill factor was about $82 \%$ due to unused edge pixels and some defective pixels. The combined Strehl ratio is $60-65 \%$, in good agreement with the measured number of $55 \%$.

In conclusion, large Strehl ratio improvements were demonstrated. For the lowest rotation speed of the phase plates, the corrected Strehl ratio of 0.55 is to be compared to an uncorrected value of 0.01 , yielding a ratio of 55 .

\section{Field tests}

For the field tests, the laboratory breadboard was moved to LLNL Site 300, a terrain of rolling hills about 15 miles southeast of LLNL. The tests were carried out across a valley, between two hills that contained the CCIT system and a retro-reflector, respectively. Figure 14 shows an elevation map below the propagation path, while Fig. 15 shows the trailer containing the CCIT system and the shack containing the retro mirrors.

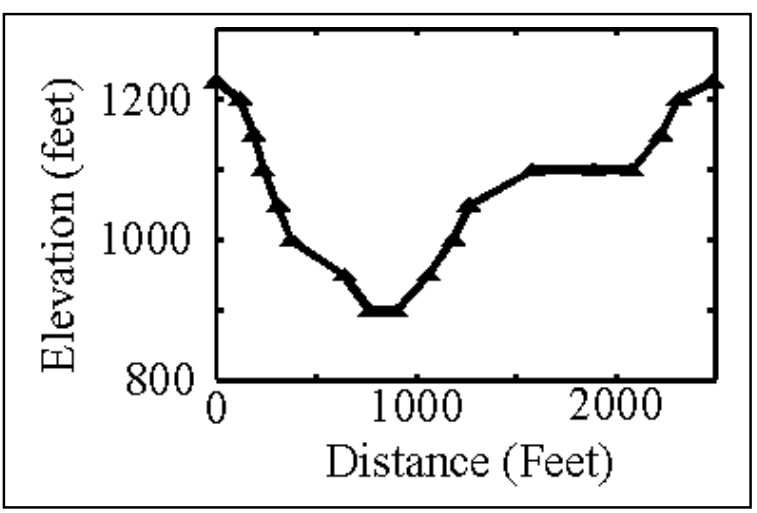

Figure 14. Elevation map below propagation path
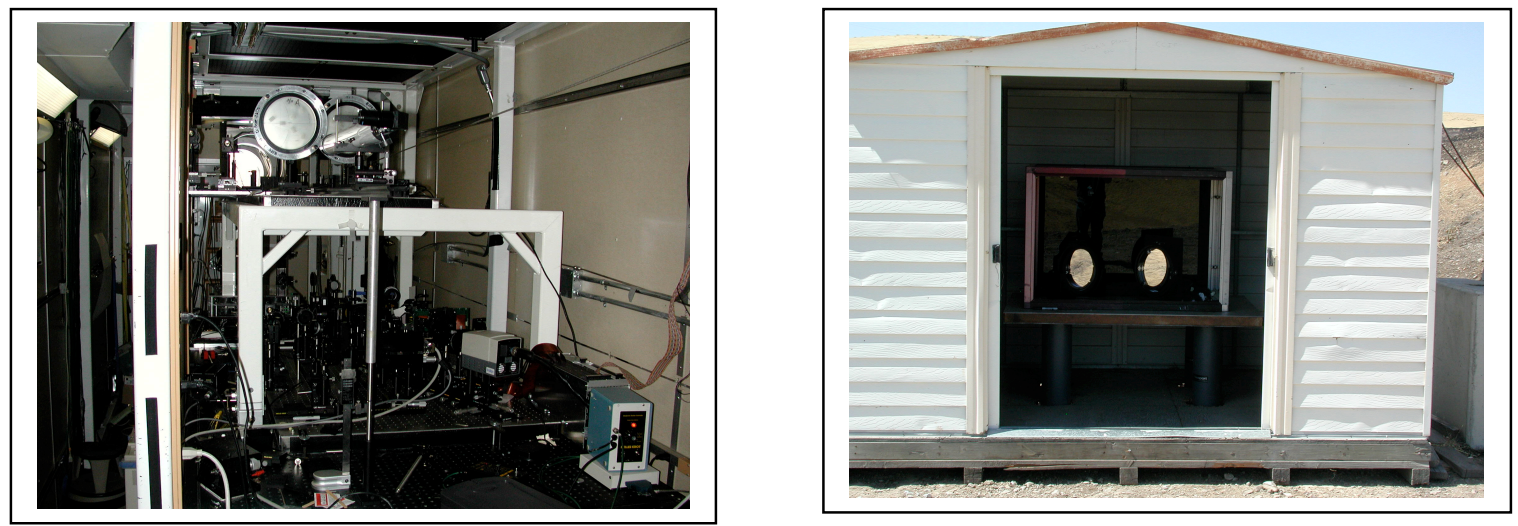

Figure 15. CCIT system mounted in trailer and shack with retro mirrors

Turbulence characterization measurements were carried out similar to the laboratory demonstrations. The measured Fried parameter was $r_{0} \sim 2.4 \mathrm{~cm}$, and the scintillation index was about 1.3 , significantly higher than for the laboratory demonstrations. Details will be provided in a separate paper.
System performance was quantified similarly to the laboratory tests. Figure 15 shows typical performance results. The corrected Strehl ratio of 0.46 is in good agreement with a projected value of $0.73 * 0.82=0.60$. The ratio of corrected to uncorrected Strehl was lower than in the laboratory demonstrations primarily because the uncorrected Strehl was higher 


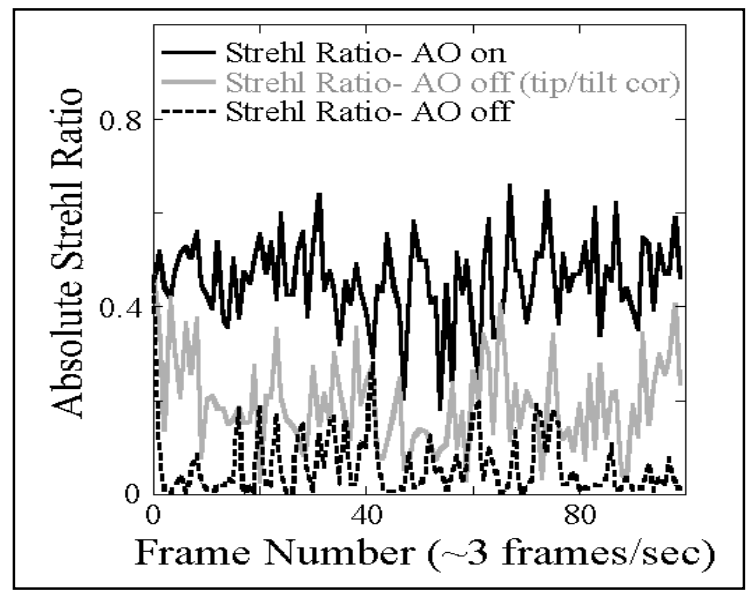

Figure 16. Field test uncorrected/corrected Strehl ratios vs. time

\section{SPATIAL LIGHT MODULATOR DEVELOPMENT}

A major objective of the CCIT program was the development of high performance SLMs, the enabling component of the holographic concept and with numerous other applications. The specifications for the piston-only Phase-I SLMs are shown in Table 1.

Table 1. Piston-only SLM specifications

\begin{tabular}{|l|c|}
\hline Parameter & Specification \\
\hline Elements $($ scalable) & $32 \times 32$ \\
\hline Stroke $(\mu \mathrm{m})$ & 0.8 \\
\hline Phase resolution $(\mathrm{bits})$ & 8 \\
\hline Response-time $(\mu \mathrm{s})$ & 10 \\
\hline Flatness $@ \lambda=1.5 \mu \mathrm{m}$ & $\lambda / 50$ \\
\hline Surface quality, rms $(\mathrm{nm})$ & 4 \\
\hline
\end{tabular}

Several parallel approaches have been pursued. They are:

- Boston Micro-Machines Corporation (BMC): Si MEMS

- Boston University: Metal MEMS

- Stanford University: Si MEMS, Silicon-oninsulator (SOI)

In addition to piston-only devices, tip-tilt-piston SLMs are being developed by Boston University/Boston MicroMachines, Stanford University, and MicroAssembly Technologies/Adriatic Research Institute.

The technical approaches are proprietary. Only a brief description of the BMC device used in the demonstrations will be given. Figure 16 shows a pixel schematic, a photograph of a section of a device, and a photograph of a packaged SLM. The actuators are based on parallel-plate capacitors. A mirror is attached to the upper plate using a post, resulting in a flat reflective surface even as the moving actuator plate bends. This device has met all of the specifications listed in Table 1. Its response was shown to be very repeatable, allowing the use of a look-up table to account for response variations across the array. For the demonstrations, the SLM was driven by electronics, built by Lucent Corporation, connected to the SLM via cables. An integrated driver is also being developed; two generations of test ASICs have been built and tested, and a final ASIC design is being fabricated for contacting to the MEMS structure. 

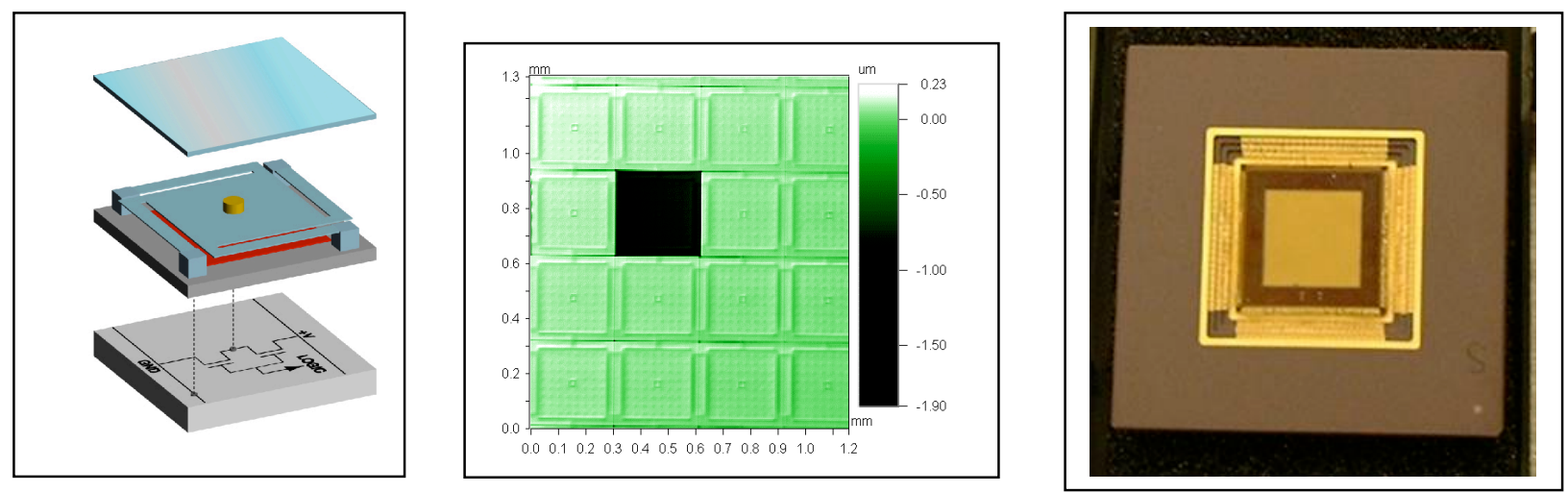

Figure 17. 32 x 32 Si SLM developed by Boston MicroMachines Corporation

Figure 18 shows measured mirror deflection versus voltage (left) and a photograph of the 32 x 32 SLM mounted in a Zygo interferometer (right).
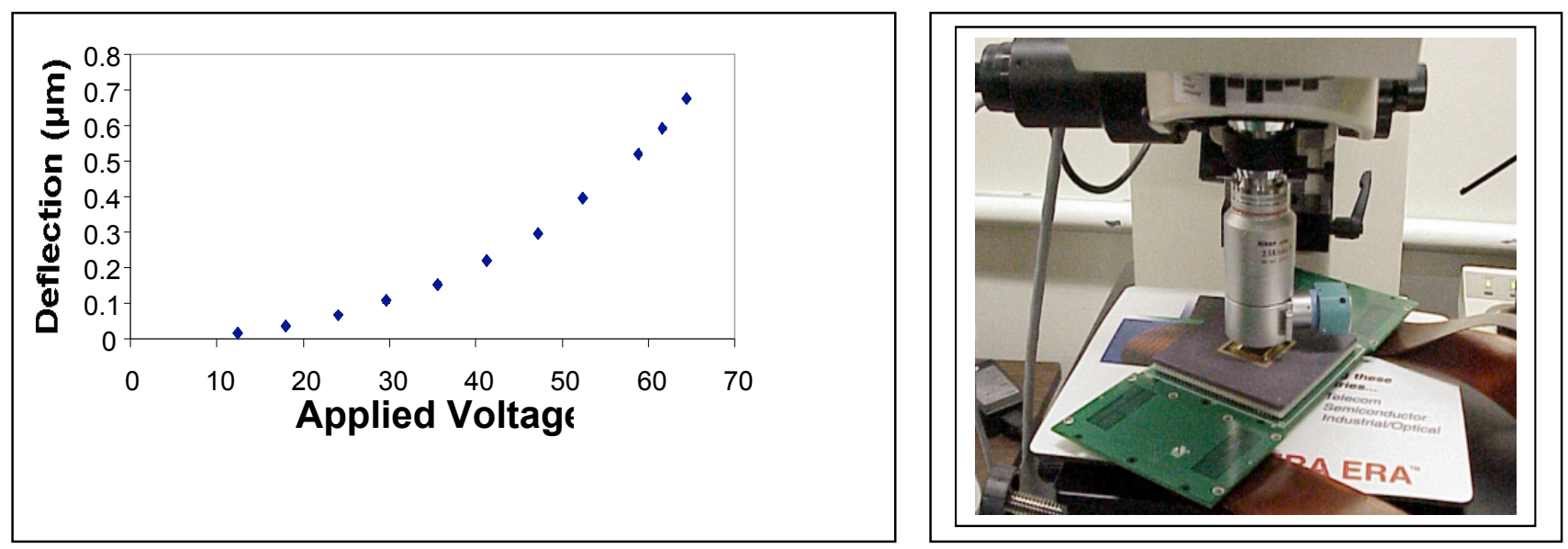

Figure 18. SLM response (left) and test SLM mounted in Zygo interferometer

\section{Conclusions}

A coherent adaptive optics concept has been demonstrated, and its application to communication systems described. Laboratory and field demonstrations of compensated receiver and conjugated transmitter configurations have been performed, and Strehl numbers of $\sim 50 \%$, in good agreement with theoretical projections, obtained. The concept provides semi-autonomous "target" acquisition and precision pointing and tracking through distorting media, at shot-noise-limited sensitivity, within a field-of-view that is proportional to the number of pixels per row/column.
Laboratory and field demonstrations have shown robust operation under conditions of significant scintillation where conventional adaptive optics systems have problems. The reasons are:

- No wavefront reconstruction is necessary,

- The coherent receiver responds to field amplitude (factor of 100 in intensity is only factor 10 in amplitude),

- The coherent receiver is insensitive to background light and electronics noise.

The concept has numerous applications in addition to communications, including imaging, sensing and precision beam delivery. 


\section{REFERENCES}

1. The CCIT concept incorporates elements of the Active Tracking System concept developed by Northrop Grumman Corporation under DARPA sponsorship. U.S. patent $5,378,888$.

2. J.H. Shapiro, "Optics of the turbulent atmosphere", Massachusetts Institute of Technology, March 1980.

3. David L. Fried, "Statistics of a geometric representation of wavefront distortion", J. Opt. Soc. Am., 1427 (1965).

4. Darryl P. Greenwood, "Bandwidth specification for adaptive optics systems", J. Opt. Soc. Am., 67, 390 (1977).

5. J. W. Hardy, Adaptive Optics for Astronomical Telescopes, Oxford University Press, Oxford, 1998.

\section{BIOGRAPHY}

Eddy A. Stappaerts received his M.S. (1972) and Ph.D. (1976) degrees from Stanford University, CA, in Electrical Engineering. He is currently with the Lawrence Livermore National Laboratory, dividing his time between the "Physics and Advanced Technologies" and the "Nonproliferation, Arms Control and International Security" Directorates. He was the program manager and chief scientist for the DARPA CCIT program. Before joining LLNL, he was Manager for Laser Technology R\&D at Northrop Grumman Corporation. His technical background is in laser and nonlinear optics devices and system applications. Dr. Stappaerts is a Senior Member of IEEE and a Member of the OSA. He has over 40 publications in professional journals and holds over 20 patents.

Kevin L. Baker received his M.S. (1991) and Ph.D. (1996) in Applied Science from the University of California, Davis. Dr. Baker has over 10 years of experience in $R \& D$ on optical diagnostics and applications. He joined the adaptive optics program at LLNL in 2001 as a staff member working on the CCIT phase I project. Before that, from 1989-2001, he worked in the field of plasma physics developing diagnostics, studying nonlinear wave-interactions in laserproduced plasmas and characterizing hohlraum dynamics and Z-Pinch physics on the $\mathrm{Z}$ accelerator. Dr. Baker is a member of the American Physical Society and the International Society for Optical Engineering. He has authored 18 publications on adaptive optics, plasma diagnostics and wave-wave interactions in nonlinear and inhomogeneous medium.

Gavel-bio

Wilks-bio
Dr. Scot S. Olivier currently leads the Adaptive Optics

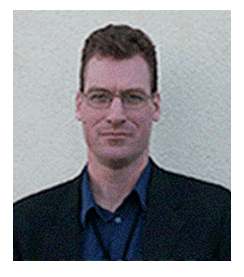

Group in the Physics and Advanced Technologies Directorate at Lawrence Livermore National Laboratory (LLNL). He joined LLNL in 1991 as a postdoctoral researcher, after receiving his Ph.D. in Physics from the University of California, Santa Cruz and his B.S. from Princeton University in Electrical Engineering and Computer Science and Engineering Physics. In 1994, he assumed a staff position as an optical physicist in the LLNL Laser Programs Directorate working as the project scientist on the Lick Observatory laser guide star adaptive optics system, which was the first system to successfully utilize a sodium-layer laser beacon. He subsequently served as the LLNL project manger for the Keck Observatory adaptive optics wavefront control and laser guide star systems. His current activities span a broad range of applications, including astronomy, human vision science, high power laser beam control, optical communications, surveillance and remote sensing. He leads several projects in the development and application of advanced adaptive optics based on optical MEMS technology. He also serves as an Associate Director of the NSF Science and Technology Center for Adaptive Optics. Dr. Olivier is a member of the SPIE, OSA, and AAS, and has published over 50 papers on adaptive optics.

Brase-bio 